\title{
Mobile modular installation for processing liquid low- and average-radioactive waste
}

\author{
V.V. Shatalov, M.Yu. Malinina and L.G. Solovyova \\ All-Russian Research Institute of Chemical Technology of Russian Federation Ministry \\ of Atomic Energy, 33 Kashirskoe Shosse, Moscow 115230, Russia
}

\begin{abstract}
The mobile modular installation for processing of liquid radioactive waste of complex physic-chemical composition is developed in co-operation of the All-Russian Research Institute of Chemical Technology and a number of research and production organisation. The complex treatment of the solutions is done in a membrane adsorption technology followed by the cementation of formed sediments and slimes. The distinctive feature of the proposed LRW processing scheme is the ability to nearly completely extract the most dangerous radionuclides Cs137, Co-60 and Sr-90 on the selective inorganic Co-, Ni-, Cu-containing adsorbents at the stage of the initial solution pre-cleaning. The developed scheme on ensures a possibility of reusing purified water according to standard OST V95.823-89 and the minimum quantity of SRW ensuing at the LRW processing.
\end{abstract}

\section{INTRODUCTION}

At present time the All-Russian Research Institute of Chemical Technology has been carrying out intensive work in the processing liquid radioactive waste (LRW) from the Russian atomic fleet. One of such activities designed within the framework of the Program of international Cupertino on environment in Arctic Region "AMEC", is the development of the mobile modular installation for processing LRW of a complex physic-chemical composition.

The mobile modular installation (MMI) is designed to treat within the shortest possible time the liquid radioactive waste accumulated and formed at the decommissioning of the temporary radioactive waste and spent-fuel storage located on the coastal and floating technical bases. It is intended for the processing LRW that can't be treated on the stationary complexes for different reasons (lack of transporttechnological scheme, difficulties in the ensuring of nuclear and environment safety, other technological and economic aspects, etc.).

The main technological problem at working on the installation was the designing of reliable secure and economical technology ensuring the processing LRW of a complex physic-chemical composition to the form that is secure for their subsequent storage at minimum quantity of a generated secondary solid radioactive waste (SRW).

\section{MAIN FEATURES AND CHARACTERISTICS OF THE INSTALLATION}

The scheme developed for the installation is based on the application of patented reliable and successfully operating Russian technologies for the processing of LRW from various radiochemical productions, including atomic power stations, civil fleet and Navy vessels $[1,2]$.

The capacity of the installation is not less than $1 \mathrm{~m}^{3} / \mathrm{h}\left(2000-2500 \mathrm{~m}^{3} /\right.$ year) of treated water (for the most composite wastewaters). The installation can process six groups of liquid radioactive waste with the characteristic listed in the Table 1.

The complex treatment of the solutions is done in a membrane adsorption technology followed by the cementation of formed sediments and slimes. The scheme provides for the final thermal conditioning of high-radioactive spent adsorbents in the special containers and brine concentrating up to dry salts in the thorough concentrating and drying apparatuses using the advanced American system of the "Ohmptemp." 
The integrated technological schemes operating in the MMI are based on the recent Russian [3] and world achievements in the LRW treatment of various kinds. They include:

- the mechanical clearing using of microfiltrating centrifuges;

- the reverse osmosis desalination with softening;

- the application of a selective adsorption of hazardous radionuclides of cesium, cobalt and strontium on $\mathrm{Ni}, \mathrm{Cu}$ - containing ferro-cyanide adsorbents and thorough decontamination of LRW on the synthetic zeolitic adsorbents based on alumosilicate [4-6];

- oxidative detoxification of LRW;

- final sanitary ion-exchange cleaning up to the standards allowing dumping into the hydrographic water system;

- the conditioning of the spent adsorbents, cementation and drying of sediments and slimes for their burial in the special containers.

The principle flow sheets for processing of non-saline and saline LRW are shown in Fig.1 and 2.

The distinctive feature of the proposed LRW processing scheme is the ability to nearly completely extract the most dangerous radionuclides $\mathrm{Cs}-137, \mathrm{Co}-60$ and $\mathrm{Sr}-90$ on the selective inorganic $\mathrm{Co}-\mathrm{Ni}$ ,Cu-containing adsorbents at the stage of the initial solution pre-cleaning. Besides, since the radionuclide concentration degree will comprise 4000 instead of 205, attainable in the salt concentration process by evaporation, then the total amount of the secondary SRW will be 2-4 times smaller, as compared with any analogous scheme without pre-cleaning stage $[7,8]$.

Such process organization of the liquid radioactive waste treatment ensures a reliable fixing of the main activity within solid adsorbents, sharply reduces the total amount of secondary solid radioactive waste to be buried, notably simplifies the LRW decontamination process and SRW utilization, improves the reliability and radiation safety.

To sum up, the developed technology that is the basis for the installation operation ensures:

- thorough decontamination of various types of liquid radioactive waste according to the requirements of NRB-99 and removal of chemical impurity up to the standards allowing dumping treated water into the natural water bodies;

- a possibility of reusing purified water according to standard OST V95.823-89;

- the minimum quantity of SRW ensuing at the LRW processing (the calculated concentration degree of radionuclides in the solid phase of adsorbents will reach $\sim 2500$, for more than 25 times exceeding analogous indices, e.g. those in a standard cleaning scheme with distilled water and a concrete block as the end product).

The scheme appears to be quite competitive with other kind due to inexpensive adsorbents used there, the simplicity and reliability of the radioactivity fixation on inorganic selective adsorbents and low waste.

Constructed on a modular type, the installation provides processing of LRW at the temperature of the ambient air from $-5^{\circ} \mathrm{C}$ to $+40^{\circ} \mathrm{C}$ and withstands seismic loads up to magnitude 6 on MSK-scale.

The installation modules have overall dimensions of the no more standard 20 -foot freight container and weight no more than 15 tons. It makes possible their transportation by seaborne, railway and motor transport directly to the places where LRW should be processed.

The assigned service life of separate modules and the installation, as a whole is 20 years.

The developed technology for processing of various LRW is 10 times cheaper than the scheme based on distillation. When some kinds of LRW are treated on the separate modules of the installation (not on the whole unit), the cost of the processing will decrease a few times more.

According to the preliminary estimation the prototype installation costs $\sim 1,200,000 \$$, the cost of processing per $1 \mathrm{~m}^{3}$ of radioactive solution is $75-300 \$$ depending on a physic-chemical structure of the waste. 


\section{CONCLUSION}

The above advanced integrated technology being a basis of the installation operation can be used both as mobile modules and stationary and is recommended as such for the decontamination of any kinds of low- and middle-level liquid radioactive waste in RF nuclear vessels and the waste in radiochemical production, at atomic power stations under operation and the decommissioning.

\section{Acknowledgements}

The authors are grateful to the scientific workers of VNIPIET (St.-Petersburg) for fruitful collaboration.

\section{References}

[1] R.A. Penzin, V.V. Milyutin, V.M.Geles and oth., "Application of Sorption Technique for Decontamination of Middle- and Low-Radioactive Waste (LRW) from radionuclides of Cesium and Strontium", Rus. Conf.on Radiochemistry, Dubna, May 17-19, 1994, p.156.

[2] A.T. Slastennikov and oth., "Application of Inorganic Adsorbents and Composed Materials for Isolation of Radionuclides from Liquid Radioactive Waste", Rus. Conf.on Radiochemistry, Dubna, May , 17-19, 1994, p.115.

[3] R.A. Penzin, V.P. Tarasov and oth., Pat. "Method for Treatment of Saline Decontaminated Waters Containing Nitrogen-Components and Heavy Metals", RU 2055814 Cl,6 C 02F 1/28,1/ 42.

[4] R.A. Penzin, N.F. Kalinin and oth., Pat. "Method for Production of Composed Ferrocyanide ddsorbents Selex-CF", RU 2007210 Cl, 5 D 01 J 20/02.

[5] V.V. Shatalov, R.A.Penzin and oth., Pat. "Method for Production of Composed Adsorbent XЖ-90", RU $2007211 \mathrm{Cl} 5$ B $01 \mathrm{~J} 20 / 02$.

[6] R.A. Penzin, V.E. Kopylov and oth., Pat. "Method for Production of Ceolyte Adsorbent of Type A", RU $2057582 \mathrm{Cl} 6$ B $01 \mathrm{~J} 20 / 18$.

[7] V.V. Shatalov, R.A. Penzin and oth., Pat. "Method for Treatment of Solutions Containing Heavy Metal Salts", RU 2055640 Cl 6 B 01 J 39/00, C 02 F 1/42.

[8] V.V. Shatalov, R.A. Penzin and oth., Pat. "Method for Decontamination of Solutions from Radionuclides", RU 2050027 Cl 6 G 21 F 9/12. 


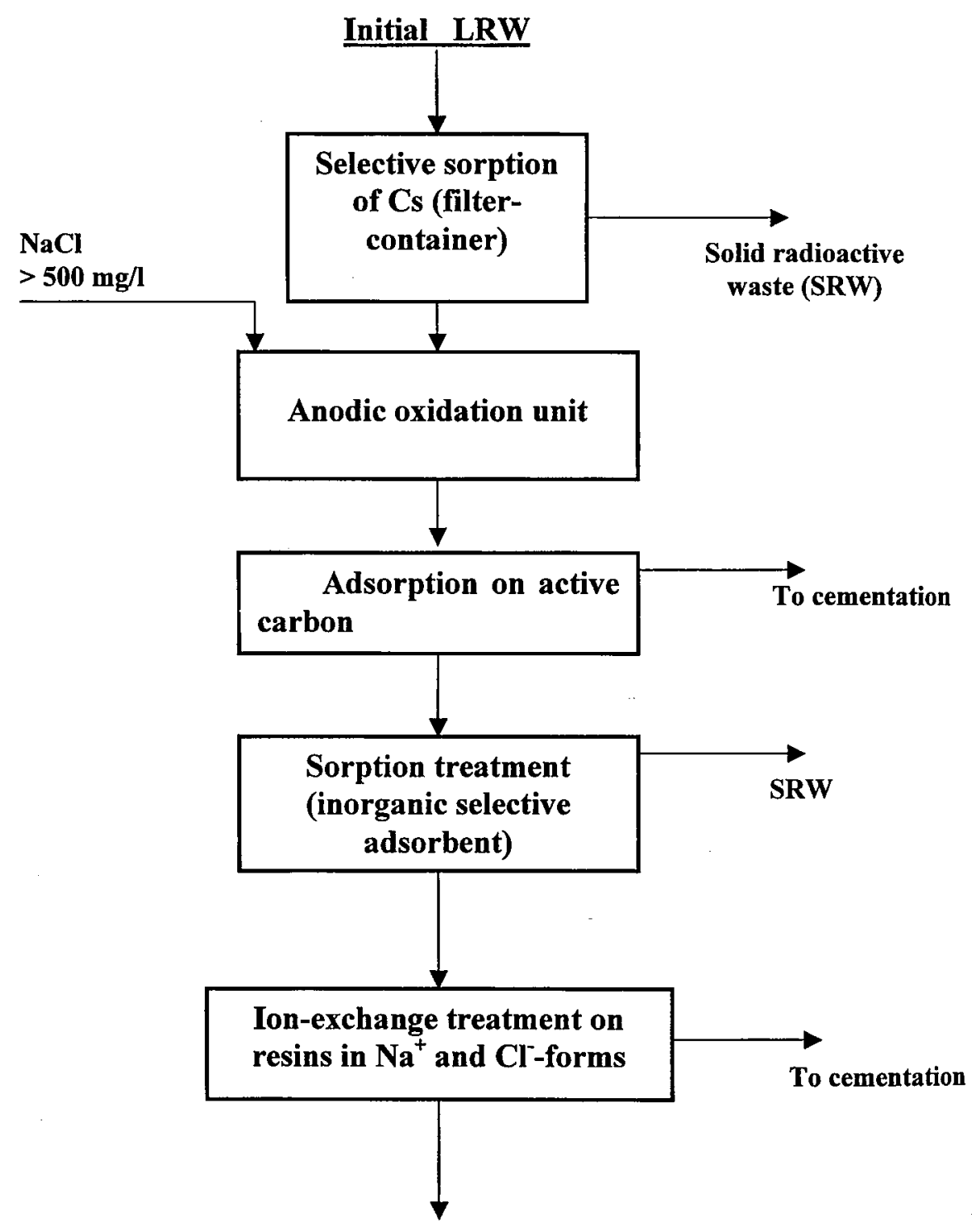

Clean water

Figure 1 : Principle flow-sheet for processing of non-saline solutions 


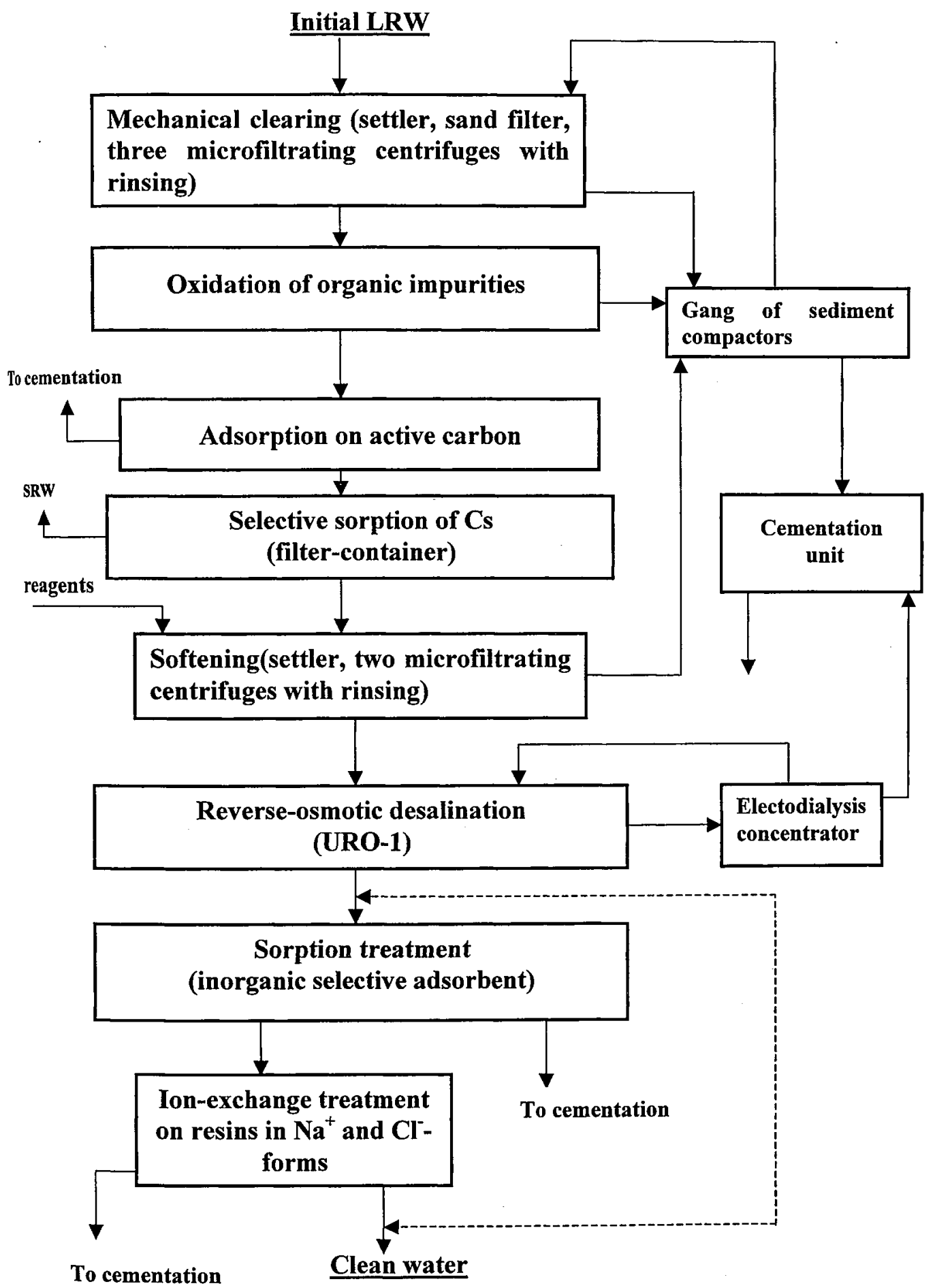

Figure 2: Principle flow-sheet for processing of saline solutions 


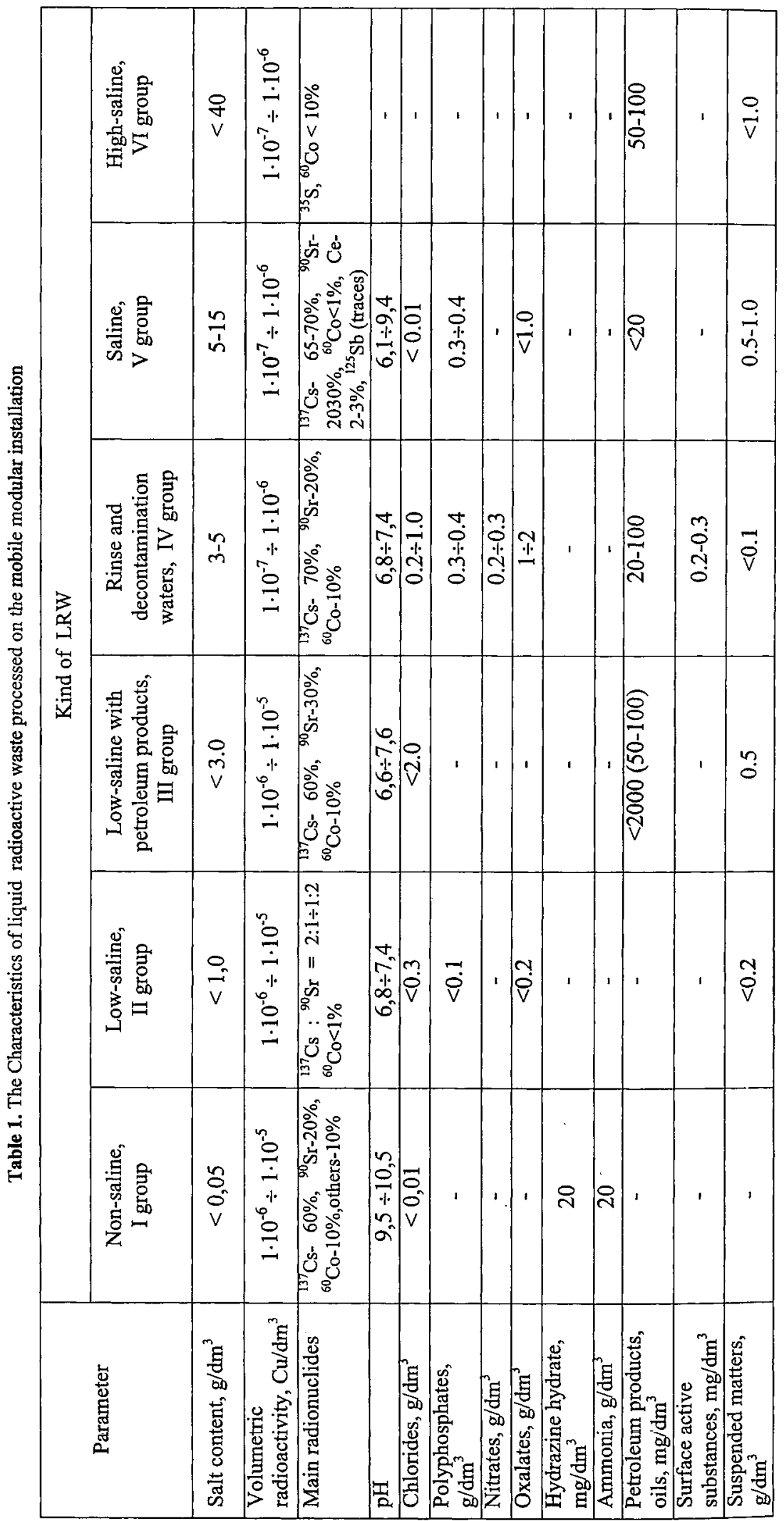

